\title{
16-dimensional compact projective planes with 3 fixed points
}

\author{
Helmut Salzmann \\ Dedicated to Professor Adriano Barlotti on the occasion of his 80 th birthday
}

Let $\mathscr{P}=(P, \mathfrak{L})$ be a topological projective plane with a compact point set $P$ of finite (covering) dimension $d=\operatorname{dim} P>0$. A systematic treatment of such planes can be found in the book Compact Projective Planes [15]. Each line $L \in \mathfrak{L}$ is homotopy equivalent to a sphere $\mathbb{S}_{\ell}$ with $\ell \mid 8$, and $d=2 \ell$, see [15] (54.11). In all known examples, $L$ is in fact homeomorphic to $\mathbb{S}_{\ell}$. Taken with the compact-open topology, the automorphism group $\Sigma=$ Aut $\mathscr{P}$ (of all continuous collineations) is a locally compact transformation group of $P$ with a countable basis, the dimension $\operatorname{dim} \Sigma$ is finite [15] (44.3 and 83.2).

The classical examples are the planes $\mathscr{P}_{\mathbb{K}}$ over the three locally compact, connected fields $\mathbb{K}$ with $\ell=\operatorname{dim} \mathbb{K}$ and the 16-dimensional Moufang plane $\mathcal{O}=\mathscr{P}_{\mathbb{O}}$ over the octonion algebra $\mathbb{D}$. If $\mathscr{P}$ is a classical plane, then Aut $\mathscr{P}$ is an almost simple Lie group of dimension $C_{\ell}$, where $C_{1}=8, C_{2}=16, C_{4}=35$, and $C_{8}=78$.

In all other cases, $\operatorname{dim} \Sigma \leqslant \frac{1}{2} C_{\ell}+1 \leqslant 5 \ell$. Planes with a group of dimension sufficiently close to $\frac{1}{2} C_{\ell}$ can be described explicitly. More precisely,

the classification program seeks to determine all pairs $(\mathscr{P}, \Delta)$, where $\Delta$ is a connected closed subgroup of Aut $\mathscr{P}$ and $b_{\ell} \leqslant \operatorname{dim} \Delta \leqslant 5 \ell$ for a suitable bound $b_{\ell} \geqslant 4 \ell-1$.

This has been accomplished for $\ell \leqslant 2$ and also for $b_{4}=17$. Here, the case $\ell=8$ will be studied; the value of $b_{\ell}$ varies with the configuration of the fixed elements of $\Delta$.

Most theorems that have been obtained so far require additional assumptions on the structure of $\Delta$. If $\operatorname{dim} \Delta \geqslant 27$, then $\Delta$ is always a Lie group [12].

By the structure theory of Lie groups, there are 3 possibilities: (i) $\Delta$ is semi-simple, or (ii) $\Delta$ contains a central torus subgroup, or (iii) $\Delta$ has a minimal normal vector subgroup, cf. [15] (94.26). The first two cases are understood fairly well:

(a) If $\Delta$ is semi-simple and $\operatorname{dim} \Delta>28$, then $\Delta \cong \mathrm{SL}_{3} \mathbb{H}$ and $\mathscr{P}$ is a Hughes plane (as described in [15] §86), or $\Delta \cong \operatorname{Spin}_{9}(\mathbb{R}, r)$ with $r \leqslant 1$, or $\mathscr{P} \cong \mathcal{O}$, see [10], [11].

(b) If $\Delta$ contains a central torus, and if $\operatorname{dim} \Delta>30$, then $\Delta^{\prime} \cong \mathrm{SL}_{3} \mathbb{H}$, see [13].

A group $\Delta$ of type (iii) fixes a point or a line, cf. [3] (XI.10.19). Hence (a) and (b) imply 
(c) If $\operatorname{dim} \Delta>30$ and $\Delta$ has no fixed element, then $\mathscr{P}$ is a Hughes plane or $\mathscr{P} \cong \mathcal{O}$.

The case that $\Delta$ fixes exactly one element has been treated in [14]:

(d) If $\operatorname{dim} \Delta \geqslant 35$ and if $\Delta$ fixes one line and no point, then $\mathscr{P}$ is a translation plane.

All such planes have been determined in [6], [7], [9]. Either $\mathscr{P} \cong \mathcal{O}$ or $\operatorname{dim} \Delta=35$.

Little progress has been made in the cases where $\Delta$ fixes exactly two elements, necessarily a point and a line. If $\operatorname{dim} \Delta \geqslant 40$, then $\mathscr{P}$ and its dual are translation planes [15] (87.7). All translation planes with $\operatorname{dim} \Delta \geqslant 38$ are described in [15] (82.28).

(e) If $\operatorname{dim} \Delta \geqslant 34$ and $\Delta$ fixes exactly 2 points and only one line, then $\Delta$ contains a translation group of dimension at least 15.

(f) If $\operatorname{dim} \Delta \geqslant 33$ and $\Delta$ fixes 2 points and 2 lines, then $\Delta$ contains a translation group $\mathrm{T} \cong \mathbb{R}^{8}$ and a compact subgroup $\Phi \cong \operatorname{Spin}_{8} \mathbb{R}$.

A method to construct all planes with exactly 2 fixed points have been given in [8].

A smaller dimension of $\Delta$ suffices if $\Delta$ fixes more than two points (the last case to be considered):

Theorem. If $\operatorname{dim} \Delta \geqslant 32$ and $\Delta$ has (at least) 3 fixed points, then $\Delta$ contains a transitive translation group $T$. Either $\operatorname{dim} \Delta=32$ and a maximal semi-simple subgroup $\Psi$ of $\Delta$ is isomorphic to $\mathrm{SU}_{4} \mathbb{C}$, or $\operatorname{dim} \Delta \geqslant 37$ and $\mathscr{P} \cong \mathcal{O}$.

Translation planes with a group $\Psi \cong \mathrm{SU}_{4} \mathbb{C}$ have already been studied in [5]. Examples of proper translation planes such that $T \Psi$ has a fixed point set $S \approx \mathbb{S}_{2}$ are given in [6].

According to the stiffness result [15] (83.23), the stabilizer $\Lambda$ of a non-degenerate quadrangle satisfies $\operatorname{dim} \Lambda \leqslant 14$. The proof of the theorem depends decisively on Bödi's improvement [1] of the stiffness theorem:

() If the fixed elements of the connected Lie group $\Lambda$ form a connected subplane $\mathscr{E}$, then $\Lambda$ is isomorphic to the 14-dimensional compact group $\mathrm{G}_{2}$ or its subgroup $\mathrm{SU}_{3} \mathbb{C}$ or $\operatorname{dim} \Lambda<8$. If $\mathscr{E}$ is a Baer subplane $(\operatorname{dim} \mathscr{E}=8)$, then $\Lambda$ is a subgroup of $\mathrm{SU}_{2} \mathbb{C}$.

Corollary. From $\operatorname{dim} \Lambda>8$ it follows that $\operatorname{dim} \mathscr{E}=2$.

Proof. Assume that $\operatorname{dim} \mathscr{E}=4$. If $L$ is any line of $\mathscr{E}$ and if $c \in L \backslash \mathscr{E}$, then $\operatorname{dim} \Lambda_{c}>0$ and the fixed elements of $\Lambda_{c}$ form a Baer subplane $\langle\mathscr{E}, c\rangle$. Hence $\operatorname{dim} \Lambda_{c} \leqslant 3$ and $\operatorname{dim} \Lambda \leqslant 11$. An alternative proof is given by [15] (96.35).

Proof of the Theorem. 1) For any closed subgroup $\Gamma \leqslant \Delta$ and any point $x$ the dimension formula $\operatorname{dim} \Gamma=\operatorname{dim} \Gamma_{x}+\operatorname{dim} x^{\Gamma}$ holds, see [15] (96.10). This fact will be used repeatedly without mention.

2) By the stiffness theorem, the stabilizer $\nabla$ of a triangle satisfies $\operatorname{dim} \nabla \leqslant 30$. Hence 
all fixed points of $\Delta$ are incident with the same line $W$. There are at least 3 fixed points $u, v, w \in W$ and the stiffness theorem implies $\operatorname{dim} \Delta \leqslant 38$.

3) Because of results (a) and (b), the group $\Delta$ has a minimal normal subgroup $\Theta \cong \mathbb{R}^{t}$. Choose $a \notin W$ and $\varrho \in \Pi \leqslant \Theta$ such that $\Pi \cong \mathbb{R}$ and $a^{\varrho} \neq a$. Since $\Delta$ acts linearly on $\Theta$, the centralizer Cs $\varrho$ is also the centralizer of $\Pi$, and the dimension formula gives $\operatorname{dim} \operatorname{Cs} \Pi \geqslant 32-t$. The connected component $\Lambda$ of $\Delta_{a} \cap$ Cs $\Pi$ fixes the orbit $a^{\Pi}$ pointwise, and the fixed elements of $\Lambda$ form a connected subplane $\mathscr{E}$, see [15] (42.1). By ( $\square$ ) we have $\operatorname{dim} \Delta_{a}-t \leqslant \operatorname{dim} \Lambda \leqslant 14$ and $t \geqslant 2 ;$ moreover, $\operatorname{dim} \Lambda=14$ or $\operatorname{dim} \Lambda \leqslant 8$.

4) Assume first that $t<8$. Then $\Lambda \cong \mathrm{G}_{2}$ is compact. Remember that the action of any compact or semi-simple Lie group on a real vector space is completely reducible ([2] (35.4)). Each irreducible module of $G_{2}$ on $\mathbb{R}^{16}$ has a dimension divisible by 7 , see [15] (95.10). Since $\Pi^{\Lambda}=\Pi$, it follows from $t \leqslant 7$ that the commutator $[\Lambda, \Theta]$ is trivial.

5) The last statement implies that the orbit $a^{\Theta}$ is contained in $\mathscr{E}$. Because $\Theta$ is commutative, $\Theta_{a}$ fixes each point of $a^{\Theta}$. Hence $\Theta_{a}$ acts trivially on the subplane $\mathscr{E}$ generated by $a^{\Pi}$ and $u, v, w$, and the connected component of $\Theta_{a}$ is contained in $\Lambda$, but $\Lambda$ is simple and $\Lambda \cap \Theta=1$. Therefore, $\operatorname{dim} \Theta_{a}=0$ and $\operatorname{dim} a^{\Theta}=t=2$.

6) Denote the connected component of $\Delta_{a}$ by $\nabla$. From steps 3 ) and 5) it follows that $\operatorname{dim} \nabla=16$. Consequently, $\nabla$ has a 2-dimensional radical $\mathrm{P}=\sqrt{\nabla}$, and $[\Lambda, \mathrm{P}]=1$. Hence $\mathscr{E}^{\mathrm{P}}=\mathscr{E}$. If $c$ is a point of $\mathscr{E}$ and $c \in a w \backslash\{a, w\}$, then $\operatorname{dim} \mathrm{P}_{c}>0$. On the other hand, $\mathrm{P}_{c}$ acts trivially on the smallest closed subplane containing $a, c, u, v$, and this subplane coincides with $\mathscr{E}$ by [15] (32.7); thus the connected component of $\mathrm{P}_{c}$ would belong to the simple group $\Lambda$. This contradiction shows that $t \geqslant 8$.

7) If $t=8$, then $16 \leqslant \operatorname{dim} \nabla=\operatorname{dim} \varrho^{\nabla}+\operatorname{dim} \Lambda \leqslant t+14=22$ and $\operatorname{dim} \Lambda \geqslant 8$. Consider the smallest closed subplane $\mathscr{F}$ containing $a^{\Theta}$ and $u, v, w$, and assume that $\mathscr{P} \neq \mathscr{F}=\mathscr{F}^{\nabla}$. Then $\nabla$ induces on $\mathscr{F}$ a group $\nabla / \mathrm{K}$ of dimension $\leqslant 7$, see [15] (83.17). Hence $\operatorname{dim} \mathrm{K} \geqslant 9$ and $\mathrm{K}$ contains $\mathrm{G}_{2}$. The Corollary implies that $\operatorname{dim} \mathscr{F}=2$ and then $\operatorname{dim} \nabla / K \leqslant 1$ and $\operatorname{dim} \mathrm{K}>14$. This contradiction shows $\mathscr{F}=\mathscr{P}$ and $\Theta_{a}=1$ (because $\Theta_{a}$ fixes $\mathscr{F}$ pointwise). By ( $\square$ ) there are two possibilities: either $\Lambda \cong \mathrm{G}_{2}$ for some $\varrho \in \Theta$, or $\Lambda \cong \mathrm{SU}_{3} \mathbb{C}$ for each choice of $\varrho$, and $\nabla$ acts transitively on $\Theta \backslash\{1\}$ by [15] (96.11). These cases will be treated separately.

8) Suppose that $\Lambda \cong G_{2}$ and that $\Lambda$ is contained in the maximal semi-simple subgroup $\Psi$ of $\Delta$. By minimality of $\Theta$ and [15] (95.6b), the group $\Psi$ acts irreducibly on $\Theta$ and $\Lambda<\Psi$. Clifford's Lemma [15] (95.5) implies that $\Lambda$ cannot be contained in a proper factor of $\Psi$, hence $\Psi$ is almost simple. Inspection of the list [15] (95.10) of representations shows that $\Psi$ is locally isomorphic to an orthogonal group. Because each action of $\mathrm{SO}_{5} \mathbb{R}$ on a compact projective plane is trivial ([15] (55.40)), the group $\Psi$ is simply connected and then $\Psi$ has a subgroup $Y \cong \operatorname{Spin}_{7} \mathbb{R}$. The central involution $\alpha \in \mathrm{Y}$ cannot be planar (or else $\mathrm{Y}$ would induce a group $\mathrm{SO}_{7} \mathbb{R}$ on the fixed plane $\left.\mathscr{F}_{\alpha}\right)$. Hence $\alpha$ is a reflection with axis $W$ and some center $c$. Because $\operatorname{dim} \Delta_{c} \leqslant 22$, we have $\operatorname{dim} c^{\Delta} \geqslant 10$ and, therefore, $\operatorname{dim} \alpha^{\Delta} \alpha \geqslant 10$. It is well-known that $\alpha^{\Delta} \alpha$ is contained in the group $\mathrm{T}$ of translations with axis $W$ and that $\alpha$ inverts each translation in $\mathrm{T}$. Consequently, $\mathrm{Y}$ acts faithfully on each invariant subgroup of $\mathrm{T}$. There is only one irreducible representation of $Y$ in dimension $\leqslant 16$, viz. the natural one on $\mathbb{R}^{8}$. It 
follows that $T \cong \mathbb{R}^{16}$ is transitive and that $\operatorname{dim} T Y=37$. Finally, [4] Satz 3.6 or [15] (81.17) shows that $\mathscr{P} \cong \mathcal{O}$.

9) Consider now the second case mentioned at the end of 7). By [15] (96.19), transitivity of $\nabla$ on $\Theta \backslash\{1\}$ implies that a maximal compact subgroup $\Phi$ of $\nabla$ is transitive on the 7-sphere $S$ consisting of all rays in $\Theta$. We know that $\mathrm{SU}_{3} \mathbb{C} \cong \Lambda<\Phi$ and that $\operatorname{dim} \Phi<\operatorname{dim} \nabla \leqslant \operatorname{dim} \Lambda+t=16$. From [15] (96.20-22) we can conclude that the commutator group $\Phi^{\prime}$ is isomorphic to $\mathrm{SU}_{4} \mathbb{C}$. Let $\omega$ denote the central involution in $\Phi^{\prime}$ and note that $\Phi^{\prime} /\langle\omega\rangle \cong \mathrm{SO}_{6} \mathbb{R}$. As in step 8), it follows that $\omega$ is a reflection with axis $W$, that the translation group $\mathrm{T}$ has dimension at least 10 , and that $\mathrm{T}$ is the sum of two 8-dimensional irreducible submodules; moreover, $\operatorname{dim} \Delta=\operatorname{dim} \nabla+\operatorname{dim} T=$ 32 , and the theorem is proved in the case $t \leqslant 8$.

10) For $t>8$, the vector group $\Theta$ contains a minimal normal subgroup $\mathrm{H} \cong \mathbb{R}^{s}$ of the connected component $\Gamma$ of $\Delta_{a v}$. Mutatis mutandis, the arguments in steps 3)-9) can be applied to $\Gamma$ and $H$ instead of $\Delta$ and $\Theta$. Using the same notation as before, we have

$$
24 \leqslant \operatorname{dim} \Gamma \leqslant \operatorname{dim} a^{\Gamma}+\operatorname{dim} \varrho^{\nabla}+\operatorname{dim} \Lambda \leqslant 8+s+\operatorname{dim} \Lambda .
$$

Hence ( $\square$ ) gives $s \geqslant 2$, moreover, $\Lambda \cong \mathrm{G}_{2}$ or $s \geqslant 8$.

11) Suppose that $s<8$. As in step 4), it follows that $[\Lambda, \mathrm{H}]=1$. Choose a point $c$ in the 2-dimensional subplane $\mathscr{E}$ with $c \in a v \backslash\{a, v\}$. Then $\operatorname{dim} c^{\mathrm{H}} \leqslant 1$ and $\mathrm{H}_{c} \cap \Lambda$ has positive dimension, but $\Lambda$ is simple. Therefore, $s \geqslant 8$. If $s=8$, the Theorem is true by the arguments 7)-9).

12) To finish the proof, let $s>8$ and consider the smallest closed subplane $\mathscr{H}$ containing $a^{\mathrm{H}}$ and $u, v, w$. If $k$ is the dimension of a line of $\mathscr{H}$, then $k \mid 8$. Note that $a^{\mathrm{H}} \subseteq a v$ and that $\mathrm{H}_{a}$ induces the identity on $\mathscr{H}$. It follows that $\operatorname{dim} \mathrm{H}_{a}>0$, hence $\mathscr{H} \neq \mathscr{P}$ and $k \leqslant 4$. Since $\mathrm{H}$ has no compact subgroups other than 1 , the stiffness theorem ( $\square$ ) shows that $\operatorname{dim} \mathrm{H}_{a}<8$, moreover, $\operatorname{dim} \mathrm{H}_{a}>3$ implies $k \leqslant 2$. Only the possibility $k=2$ remains. By [15] (55.4), each closed subplane of $\mathscr{H}$ is connected, and $\mathscr{H}^{\nabla}=\mathscr{H}$ because $\mathrm{H}$ is normal in $\Gamma$. There are points $b, c \in a v \cap \mathscr{H}$ such that $\nabla_{b, c}$ fixes $\mathscr{H}$ pointwise. On the other hand, $\operatorname{dim} \nabla_{b, c} \geqslant 12$. This contradicts the Corollary.

\section{References}

[1] R. Bödi, On the dimensions of automorphism groups of eight-dimensional ternary fields. II. Geom. Dedicata 53 (1994), 201-216. MR 96c:51028 Zbl 0829.51007

[2] H. Freudenthal, H. de Vries, Linear Lie groups. Academic Press 1969. MR 41 \#5546 Zbl 0377.22001

[3] T. Grundhöfer, H. Salzmann, Locally compact double loops and ternary fields. In: Quasigroups and loops: theory and applications, 313-355, Heldermann, Berlin 1990. MR 93g:20133 Zbl 0749.51016

[4] H. Hähl, Lokalkompakte zusammenhängende Translationsebenen mit großen Sphärenbahnen auf der Translationsachse. Resultate Math. 2 (1979), 62-87. MR 82a:51010 Zbl 0437.51011

[5] $\mathrm{H}$. Hähl, $\mathrm{SU}_{4}(\mathbb{C})$ als Kollineationsgruppe in sechzehndimensionalen lokalkompakten Translationsebenen. Geom. Dedicata 23 (1987), 319-345. MR 88j:51023 Zbl 0622.51008 
[6] $\mathrm{H}$. Hähl, Sixteen-dimensional locally compact translation planes admitting $\mathrm{SU}_{4} \mathbb{C} \cdot \mathrm{SU}_{2} \mathbb{C}$ or $\mathrm{SU}_{4} \mathbb{C} \cdot \mathrm{SL}_{2} \mathbb{R}$ as a group of collineations. Abh. Math. Sem. Univ. Hamburg 70 (2000), 137-163. MR 2003b:51023 Zbl 0992.51007

[7] H. Hähl, Sixteen-dimensional locally compact translation planes with large automorphism groups having no fixed points. Geom. Dedicata 83 (2000), 105-117. MR 2001h:51021 Zbl 0973.51011

[8] H. Hähl, H. Salzmann, 16-dimensional compact projective planes with a large group of automorphisms fixing two points. In preparation.

[9] $\mathrm{H}$. Löwe, 16-dimensional locally compact, connected translation planes admitting $\mathrm{SL}_{2} \mathbb{H}$ as a group of collineations. To appear in Pacific J. Math. 209 (2003), 325-337.

[10] B. Priwitzer, Large semisimple groups on 16-dimensional compact projective planes are almost simple. Arch. Math. (Basel) 68 (1997), 430-440. MR 98e:51020 Zbl 0877.51014

[11] B. Priwitzer, Large almost simple groups acting on 16-dimensional compact projective planes. Monatsh. Math. 127 (1999), 67-82. MR 2000d:51020 Zbl 0929.51010

[12] B. Priwitzer, H. Salzmann, Large automorphism groups of 16-dimensional planes are Lie groups. J. Lie Theory 8 (1998), 83-93. MR 99f:51027 Zbl 0902.51012

[13] H. Salzmann, Characterization of 16-dimensional Hughes planes. Arch. Math. (Basel) 71 (1998), 249-256. MR 99i:51013 Zbl 0926.51016

[14] H. Salzmann, Near-homogeneous 16-dimensional planes. Adv. Geom. 1 (2001), 145-155. MR 2002h:51009 Zbl 1002.51011

[15] H. Salzmann, D. Betten, T. Grundhöfer, H. Hähl, R. Löwen, M. Stroppel, Compact projective planes. de Gruyter 1995. MR 97b:51009 Zbl 0851.51003

Received 28 August, 2002

H. Salzmann, Mathematisches Institut der Universität Tübingen, Auf der Morgenstelle 10, 72076 Tübingen, Germany

Email: helmut.salzmann@uni-tuebingen.de 